\title{
Reduced fibrillar collagen accumulation in skeletal muscle of secreted protein acidic and rich in cysteine (SPARC)-null mice
}

\author{
Sanae OMI ${ }^{1)}$, Keitaro YAMANOUCHI ${ }^{1) *}$, Katsuyuki NAKAMURA ${ }^{1)}$, \\ Takashi MATSUWAKI ${ }^{1)}$ and Masugi NISHIHARA ${ }^{1)}$ \\ 1)Department of Veterinary Physiology, Graduate School of Agricultural and Life Sciences, The University of \\ Tokyo, 1-1-1 Yayoi, Bunkyo-ku, Tokyo 113-8657, Japan
}

\section{J. Vet. Med. Sci.}

81(11): 1649-1654, 2019

doi: 10.1292/jvms.19-0485

Received: 30 August 2019

Accepted: 14 September 2019

Advanced Epub:

2 October 2019
ABSTARCT. We have previously shown that secreted protein acidic and rich in cysteine (SPARC) promotes myogenic differentiation of rat skeletal muscle progenitor cells in vitro, and in vivo small interfering RNA (siRNA)-mediated transient suppression of SPARC expression in skeletal muscle of mice causes atrophic changes of myofibers, suggesting that SPARC plays a role in the maintenance of skeletal muscle function. In order to know the effect of long-term deficiency of SPARC on skeletal muscle, we performed phenotypic analyses of skeletal muscle of SPARC-null mice. Age-associated changes of myofiber diameters were comparable between wild type (WT) and SPARC-null mice at all ages examined, indicating that the growth of myofibers is unaffected by the absence of SPARC. On the other hand, accumulation of fibrillar collagen was significantly reduced in SPARC-null mice compared to WT mice after 5 months of age without significant changes of collagen I gene expression. The results obtained in the present study suggest that SPARC plays a role to maintain the stiffness of skeletal muscle by regulating collagen accumulation.

KEY WORDS: collagen, fibrosis, myofiber, skeletal muscle, Secreted protein acidic and rich in cysteine

Skeletal muscle comprises a bundle of myofibers, which are terminally differentiated multinucleated cells. The stiffness of skeletal muscle is affected by a variety of factors. The composition of extracellular matrix (ECM), especially, fibrillar collagen, is the major factor affecting skeletal muscle stiffness [19]. Type I collagen is the most abundant collagen species comprising fibrillar collagen, while type IV collagen is the major constituent of basement membrane [8].

Secreted protein acidic and rich in cysteine (SPARC) is a secretory nonstructural, matricellular glycoprotein. We have previously shown that SPARC promotes myogenic differentiation of rat skeletal muscle progenitor cells in vitro [15]. In addition, siRNAmediated transient suppression of SPARC expression in skeletal muscle of mice caused atrophic changes of myofibers [14]. These results suggested that SPARC plays a role in the maintenance of skeletal muscle function.

SPARC is shown to be involved in the regulation of fibrillar collagen synthesis in heart and skin [3, 6]. Pro-collagen bound by SPARC is hindered from interacting with surface collagen receptors, thus leading to an efficient incorporation into fibrillar collagen. However, in the absence of SPARC, as pro-collagen is retained on the cell surface receptor, fibrillar collagen synthesis is suppressed [17]. In accordance with this mechanism, phenotypic analyses of SPARC-null mice revealed that several connective tissues such as those in the dermis, heart and fat were shown to contain less fibrillar collagen [2]. Fibrosis, an excessive accumulation of fibrillar collagen, in skeletal muscle impairs its function [11, 12], and is a cause of increased stiffness of skeletal muscle. Thus, elucidating whether SPARC is also involved in the regulation of fibrillar collagen accumulation in skeletal muscle is important to understand the mechanism of fibrosis, which is a hallmark of some skeletal muscle pathologies such as muscular dystrophy and sarcopenia (age-associated skeletal muscle loss).

In order to know the effect of long-term deficiency of SPARC on skeletal muscle phenotype, in the present study, we employed SPARC-null mice and performed phenotypic analyses of skeletal muscle.

\section{MATERIALS AND METHODS}

\section{Mice and sample collection}

A pair of male and female SPARC-(+/-) C57BL/6J mice was kindly gifted from Dr. Amy D. Bradshaw [16]. They were inhouse crossed, and the PCR-genotyped off-springs were bred for several generations. Only wild type (WT) and SPARC-(-/-) 
(SPARC-null) male mice were used in this study. All mice were maintained in the animal facility of our laboratory under controlled environmental conditions at $23^{\circ} \mathrm{C}$ with lighting conditions of $12 \mathrm{hr}$ of light and $12 \mathrm{hr}$ of darkness (lights on at 0700). Mice were fed ad libitum with commercial chow (Lab MR-Stock Nihon Nosan Kogyo, Yokohama, Japan). Mice were killed by inhalation of carbon dioxide gas at 2, 5, 11, 16 and 22 months of age, and the tibialis anterior (TA) muscle was obtained. TA muscles were then snap-frozen in isopentane cooled by liquid nitrogen and stored at $-80^{\circ} \mathrm{C}$ until use. The number of mice used were as follows: 2 months (WT=4, SPARC-null=4), 5 months (WT=3, SPARC-null=4), 11 months (WT=3, SPARC-null=4), 16 months (WT=3, SPARC-null=9), and 22 months (WT=5, SPARC-null=3), respectively. All animal experiments were performed in accordance with the Guide for the Care and Use of Laboratory Animals of the University of Tokyo and were approved by the Institutional Animal Care and Use Committee of The University of Tokyo.

\section{Histological analyses}

Frozen sections ( $7 \mu \mathrm{m}$ thick) were prepared transversely from mid-belly region of TA muscle by a cryostat (Microm International GmbH, Walldolf, Germany) and attached on Matsunami Adhesive Silane (MAS)-coated slide glass (Matsunami Glass Ind., Ltd., Osaka, Japan). One section from each muscle was used for the following analyses.

The sections were fixed with $4 \%$ paraformaldehyde in phosphate buffered saline (PFA/PBS) for 15 min at room temperature. After washing thrice with PBS (5 min each), the sections were stained with Masson trichrome solution, dehydrated, and mounted with Multi Mount 220 (Matsunami Glass Ind., Ltd.).

For immunohistochemistry, the sections were fixed with 4\% PFA/PBS for $15 \mathrm{~min}$ at room temperature, washed 3 times (5 min each) with PBS, and blocked with 5\% normal goat serum (NGS) in PBS for $30 \mathrm{~min}$. After blocking, the sections were incubated at $4{ }^{\circ} \mathrm{C}$ overnight with rabbit anti-laminin antibody (Sigma; 1:200 with 5\% NGS/PBS). After washing thrice with PBS (5 min each), the sections were incubated with AlexaFluor 488-conjugated secondary antibody (Invitrogen; 1:500 with 5\% NGS/PBS) for 1 hr at room temperature. Nuclei were counterstained with Hoechst 33258, and mounted with Fluoromount ${ }^{\mathrm{TM}}$ (Diagnostic Biosystems, Pleasanton, CA, U.S.A.).

For myofiber size analysis, laminin-stained sections were observed and photographed randomly under a microscope (BX51; Olympus) with $20 \times$ objective so that at least 200 fibers were analyzed. The images were converted to binary images with Adobe Photoshop Elements 10 (2) (Adobe Systems Inc., San Jose, CA, U.S.A.), and minimum feret diameters of myofibers surrounded by laminin-staining were measured with Image J software (ver 1.46r; NIH, Bethesda, MD, U.S.A.). For analysis of Masson trichromestained area, seven randomly chosen images for each section were taken with $10 \times$ objective, and converted to binary images. The converted images were analyzed with Image J software to calculate Masson trichrome-stained area.

\section{Quantitative real time PCR}

The TA muscles were homogenized in TRIzol (Invitrogen, Carlsbad, CA, U.S.A.) using Shake Master (ver. 1.0, Bio Medical Science Inc., Tokyo, Japan), and RNA was isolated according to the manufacturer's protocol. The RNA was reverse transcribed to cDNA using SuperScript II (Invitrogen). The Thunderbird SYBR green qPCR system (TOYOBO, Osaka, Japan) was used to analyze the expression of genes detected on the Light Cycler (Roche, Basel, Switzerland). The primer set used were as follows: collA1 forward, ccgctggtcaagatggtc; collA1 reverse, ctccagaattccaggttct; col4A1 forward, tggcacaaaagggacgag; col4A1 reverse, ggccaggaataccaggaag; hprt forward, agtcccagcgtcgtgattagccat; hprt reverse, cttgagcacacagagggccacaat.

\section{Statistical analysis}

Values are shown as mean and standard error. A Student $t$-test was used to examine statistical differences between 2 groups. Both mean and median values of myofiber diameters were evaluated as follows. Mean values were compared using the Student $t$-test, whereas median values were compared using the Wilcoxon rank sum test. Statistical analyses were performed with Statcel 3 (add-in software for Excel from The Publisher OMS Ltd., Tokyo, Japan). P-values less than 0.05 were considered statistically significant.

\section{RESULTS}

Age-related changes in overall body and skeletal muscle (tibialis anterior) weights are shown in Fig. 1A. After 5 months of age, the body weights of SPARC-null mice were lower compared to WT mice though the statistically significant differences were observed $(P<0.05)$ only at 5 and 11 months of age. The skeletal muscle weights of SPARC-null mice were also lower than WT mice after 2 months of age though the statistically significant difference was observed $(P<0.05)$ only at 5 months of age (Fig. 1B). These results suggest that the overall decrease in whole body weights of SPARC-null mice reflect, at least in part, the reduced skeletal muscle weights.

In order to know whether decreased skeletal muscle weights of SPARC-null are due to the growth retardation of myofibers, the age-related changes in the diameters of myofibers were measured. As shown in Fig. 2, the distributions of myofiber diameters were comparable between WT- and SPARC-null mice at all ages examined, indicating that the growth of myofibers is unaffected by the absence of SPARC.

Since SPARC is involved in the regulation of fibrillar collagen synthesis [3, 6, 17], and several connective tissues such as those in the dermis, heart and fat were shown to contain less fibrillar collagen in SPARC-null mice [2, 4, 6, 10], it is possible that the accumulation of collagen fibril in skeletal muscle was altered in the absence of SPARC, reflecting in the reduced skeletal muscle 
A

Body weight (g)

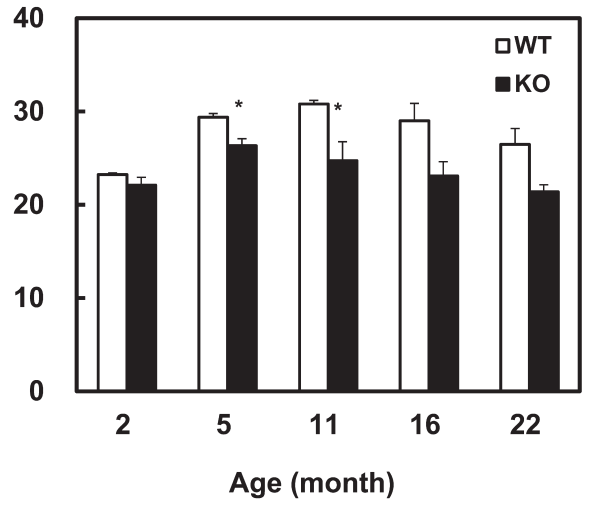

B

TA muscle weight (g)

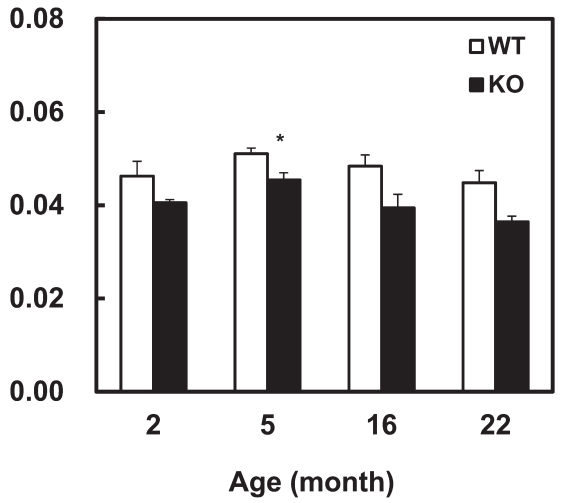

Fig. 1. Body (A) and tibialis anterior (TA) muscle (B) weights of wild-type (WT) and secreted protein acidic and rich in cysteine (SPARC)null (KO) mice. Underlined text above the graph indicates the label on the Y-axis. Data are means \pm SEM (n=3-9). $* P<0.05$ vs WT mice.

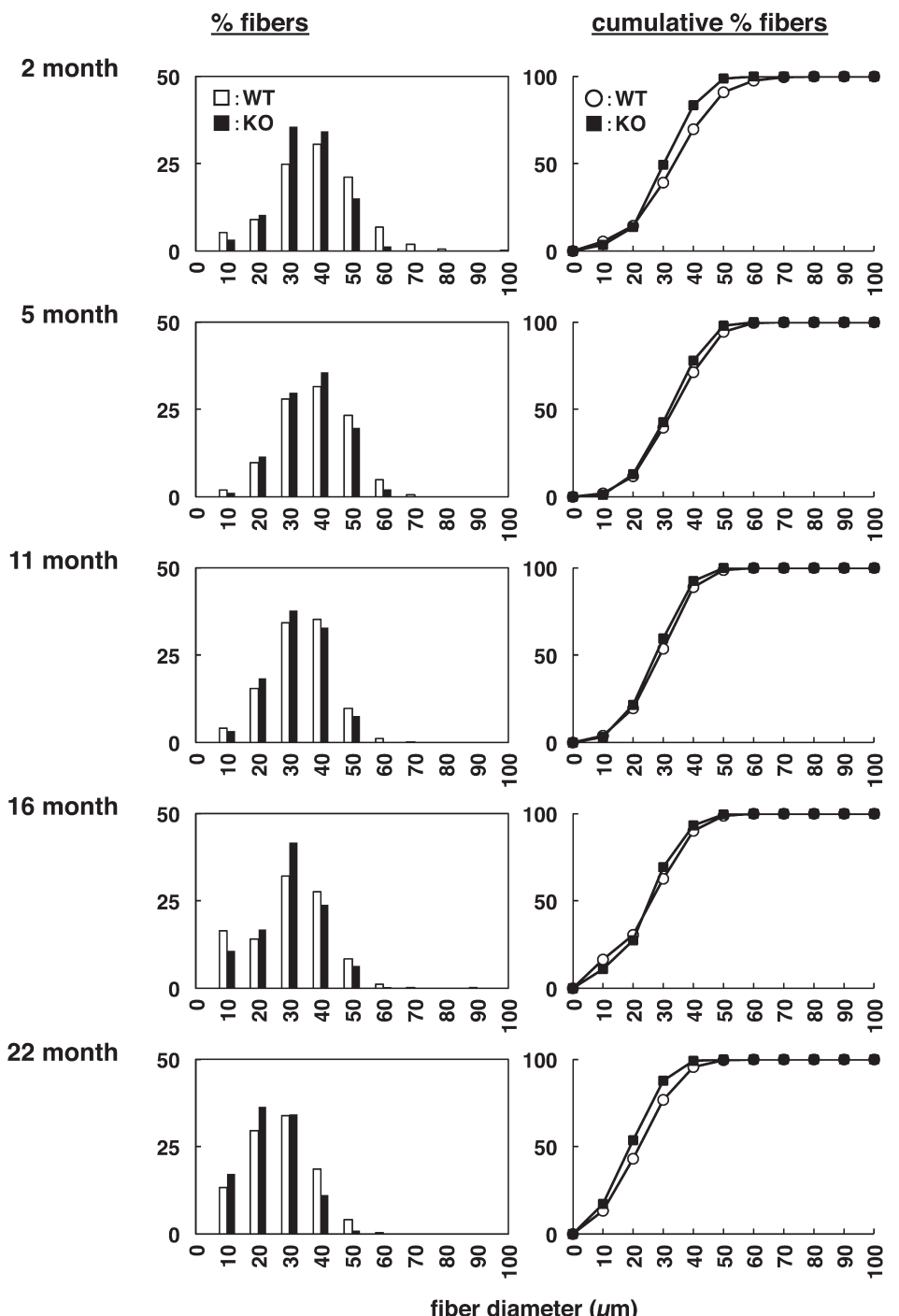

Fig. 2. Distribution of myofiber diameters of tibialis anterior (TA) muscle of wild-type (WT) and SPARC-null (KO) mice (n=3-9). The left panel depicts the relative distribution of myofibers with certain diameters. The right panel depicts relative cumulative plots based on the data shown in the left panel. White and black symbols in the right panel are WT and KO mice, respectively. Underlined text above the graph indicates the label on the Y-axis. 
weights of SPARC-null mice. As depicted in Fig. 3, quantitative analyses of Masson trichrome-stained area revealed that the accumulation of collagen fibril was significantly reduced in SPARC-null mice compared to WT mice after 5 months of age (Fig. 3B). These results suggest that the lack of SPARC leads to a decreased fibrillar collegen accumulation in skeletal muscle.

The major fibrillar collagen species in a variety of tissues including skeletal muscle is type I collagen [11]. In order to know whether the reduced collagen fibril in skeletal muscle of SPARC-null mice is accompanied with decreased type I collagen gene expression, qRT-PCR was performed. As shown in Fig. 4A, the expression level of collAl (corresponding to type I collagen) was comparable between WT and SPARC-null mice, except at 22 months of age. In addition, there was no difference in col4A1 (corresponding to type IV non-fibrillar collagen) expression between WT and SPARC-null mice. These results indicated that, at least up to 16 months of age, the reduced fibrillar collagen accumulation in skeletal muscle of SPARC-null mice is not due to decreased gene expression.

\section{DISCUSSION}

The current study demonstrated that the lack of SPARC does not affect growth of myofibers but leads to reduced accumulation of collagen fibril independent of type I collagen (collA1) gene expression.

We reported previously that transient inhibition of SPARC expression in mouse skeletal muscle by in vivo siRNA treatment causes myofiber atrophy, which is accompanied with an up-regulation of atrogin1, skeletal muscle specific ubiquitin ligase, and suggested that SPARC is involved in maintaining skeletal muscle mass [14]. We also demonstrated that siRNA-mediated suppression of SPARC expression inhibited myogenic differentiation of rat skeletal muscle progenitor cells in vitro [15]. On the other hand, in the present study, we did not observe any difference in the myofiber growth between WT and SPARC-null mice at all ages examined. Though the discrepancy between our two studies is currently unclear, it should be noted that the SPARC family of proteins, such as hevin, SMOC-1, SMOC-2, SPOCKs, and follistatin-like protein 1, are not only structurally similar to SPARC but also have partially redundant functions [2], and most of SPARC family of proteins is shown to be present in skeletal muscle [5, 7, $9,10]$. Thus, it is possible that the other SPARC family of proteins compensates continuous absence of SPARC.

SPARC has a role in post-synthetic pro-collagen processing $[3,6]$ including incorporation and stabilization of collagen I into the insoluble ECM [17]. It is reported that increased myocardial fibrosis is accompanied with the preceding up-regulation of SPARC expression, and this increased fibrosis is not correlated with the expression level of collA1 [13]. In addition, the absence of SPARC resulted in an increased cardiac rupture after acute myocardial infarction due to an insufficient accumulation of assembled fibrillar collagen [18]. The experimentally induced liver fibrosis has been reduced in SPARC-null mice compared with WT mice [1]. These reports are in accordance with our current results that accumulation of fibrillar collagen in skeletal muscle of SPARC-null mice is less than WT mice, and reduced accumulation of fibrillar collagen was observed without decreased collA1 expression, except at 22

A
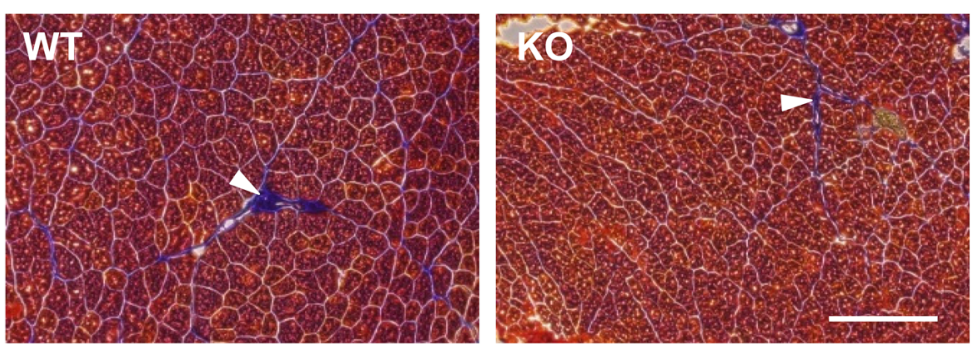

B

\section{Area (pixel x 105)}


Fig. 3. Fibrillar collagen accumulation in tibialis anterior (TA) muscle of wild-type (WT) and SPARC-null (KO) mice. Panel A depicts images of representative Masson trichrome staining. Stained area was indicated by arrowheads. Graphed data in B are quantitative analysis of area stained with Masson trichrome. Underlined text above the graph indicates the label on the Y-axis. Data are means $\pm \operatorname{SEM}(\mathrm{n}=3-9)$. Bar $=100 \mu \mathrm{m} . * P<0.05$ vs WT mice. 
A

\section{col1A1/hprt}
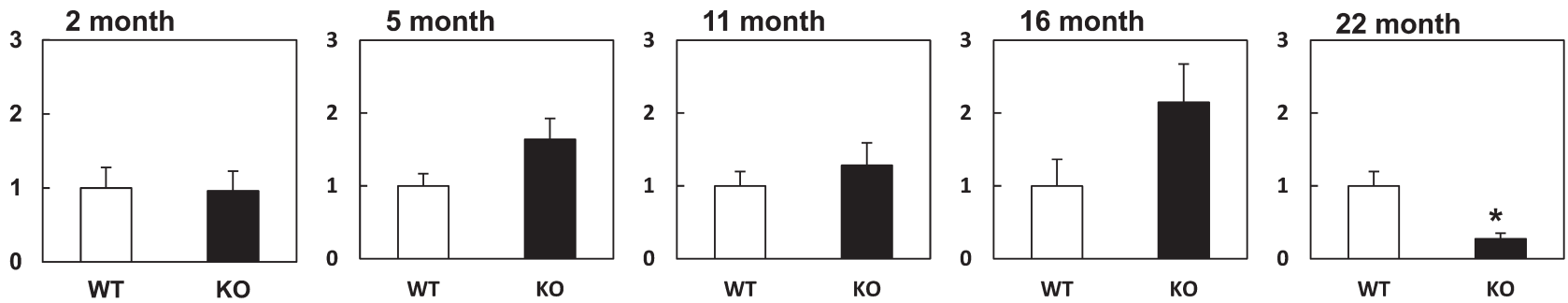

B

\section{col4A1/hprt}
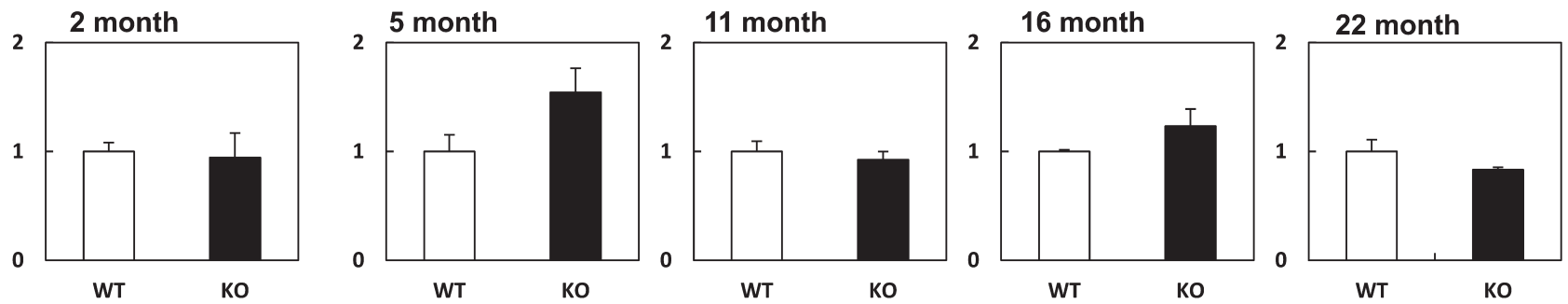

Fig. 4. Quantification of mRNA levels of collA1 (A) and col4A1 (B) in tibialis anterior (TA) muscle of wild-type (WT) and SPARC-null (KO) mice. Data are normalized by dividing with hprt expression level, and the ratios to values of WT are graphed. Underlined text above the graph indicates the label on the Y-axis. Data are means \pm SEM $(\mathrm{n}=3-9)$. ${ }^{*} P<0.05$ vs WT mice.

months of age. Thus, it is suggested that SPARC has a role in the regulation of fibrillar collagen content in extracellular matrix, and SPARC would be a possible therapeutic target to reduce fibrosis in muscular dystrophy and sarcopenia.

Myocardial stiffness and the amount of accumulating fibrillar collagen content are well correlated $[3,13]$. It should be noted that, in the present study, although no quantitative analysis was performed, the experimenters (the first and corresponding authors) noticed at the time of sampling that the skeletal muscle of SPARC-null mice is softer than WT mice. Since the stiffness is one of the important factors affecting meat quality, reducing fibrillar collagen content in skeletal muscle would possibly lead to an increased tenderness of meat. Thus, SPARC is expected to be a possible target molecule to control meat quality in animal science field.

ACKNOWLEDGMENTS. The authors are grateful to Dr. Amy D. Bradshaw, Medical University of South Carolina, for providing us a pair of SPARC-(+/-) C57BL/6J mice. This study was supported by the Japan Society for the Promotion of Science KAKENHI Grants $16 \mathrm{H} 05041$ and grant from the Ito Foundation to KY.

\section{REFERENCES}

1. Atorrasagasti, C., Peixoto, E., Aquino, J. B., Kippes, N., Malvicini, M., Alaniz, L., Garcia, M., Piccioni, F., Fiore, E. J., Bayo, J., Bataller, R., Guruceaga, E., Corrales, F., Podhajcer, O. and Mazzolini, G. 2013. Lack of the matricellular protein SPARC (secreted protein, acidic and rich in cysteine) attenuates liver fibrogenesis in mice. PLoS One 8: e54962. [Medline] [CrossRef]

2. Bradshaw, A. D. 2012. Diverse biological functions of the SPARC family of proteins. Int. J. Biochem. Cell Biol. 44: 480-488. [Medline] [CrossRef]

3. Bradshaw, A. D., Baicu, C. F., Rentz, T. J., Van Laer, A. O., Bonnema, D. D. and Zile, M. R. 2010. Age-dependent alterations in fibrillar collagen content and myocardial diastolic function: role of SPARC in post-synthetic procollagen processing. Am. J. Physiol. Heart Circ. Physiol. 298: H614H622. [Medline] [CrossRef]

4. Bradshaw, A. D., Graves, D. C., Motamed, K. and Sage, E. H. 2003. SPARC-null mice exhibit increased adiposity without significant differences in overall body weight. Proc. Natl. Acad. Sci. U.S.A. 100: 6045-6050. [Medline] [CrossRef]

5. Brekken, R. A., Sullivan, M. M., Workman, G., Bradshaw, A. D., Carbon, J., Siadak, A., Murri, C., Framson, P. E. and Sage, E. H. 2004. Expression and characterization of murine hevin (SC1), a member of the SPARC family of matricellular proteins. J. Histochem. Cytochem. 52: 735-748. [Medline] [CrossRef]

6. Card, L., Henderson, N., Zhang, Y., Bornstein, P. and Bradshaw, A. D. 2010. Expression in SPARC-null mice of collagen type I lacking the globular domain of the $\alpha 1$ (I) N-propeptide results in abdominal hernias and loss of dermal collagen. Matrix Biol. 29: 559-564. [Medline] [CrossRef]

7. Cifuentes-Diaz, C., Alliel, P. M., Charbonnier, F., de la Porte, S., Molgó, J., Goudou, D., Rieger, F. and Périn, J. P. 2000. Regulated expression of the proteoglycan SPOCK in the neuromuscular system. Mech. Dev. 94: 277-282. [Medline] [CrossRef]

8. Exposito, J. Y., Valcourt, U., Cluzel, C. and Lethias, C. 2010. The fibrillar collagen family. Int. J. Mol. Sci. 11: 407-426. [Medline] [CrossRef]

9. Gersdorff, N., Müller, M., Schall, A. and Miosge, N. 2006. Secreted modular calcium-binding protein-1 localization during mouse embryogenesis. 
Histochem. Cell Biol. 126: 705-712. [Medline] [CrossRef]

10. Görgens, S. W., Raschke, S., Holven, K. B., Jensen, J., Eckardt, K. and Eckel, J. 2013. Regulation of follistatin-like protein 1 expression and secretion in primary human skeletal muscle cells. Arch. Physiol. Biochem. 119: 75-80. [Medline] [CrossRef]

11. Lieber, R. L. and Ward, S. R. 2013. Cellular mechanisms of tissue fibrosis. 4. Structural and functional consequences of skeletal muscle fibrosis. Am. J. Physiol. Cell Physiol. 305: C241-C252. [Medline] [CrossRef]

12. Mahdy, M. A. A. 2019. Skeletal muscle fibrosis: an overview. Cell Tissue Res. 375: 575-588. [Medline] [CrossRef]

13. McDonald, L. T., Zile, M. R., Zhang, Y., Van Laer, A. O., Baicu, C. F., Stroud, R. E., Jones, J. A., LaRue, A. C. and Bradshaw, A. D. 2018. Increased macrophage-derived SPARC precedes collagen deposition in myocardial fibrosis. Am. J. Physiol. Heart Circ. Physiol. 315: H92-H100. [Medline] [CrossRef]

14. Nakamura, K., Nakano, S., Miyoshi, T., Yamanouchi, K. and Nishihara, M. 2013. Loss of SPARC in mouse skeletal muscle causes myofiber atrophy. Muscle Nerve 48: 791-799. [Medline] [CrossRef]

15. Nakamura, K., Nakano, S., Miyoshi, T., Yamanouchi, K., Matsuwaki, T. and Nishihara, M. 2012. Age-related resistance of skeletal muscle-derived progenitor cells to SPARC may explain a shift from myogenesis to adipogenesis. Aging (Albany NY) 4: 40-48. [Medline] [CrossRef]

16. Norose, K., Clark, J. I., Syed, N. A., Basu, A., Heber-Katz, E., Sage, E. H. and Howe, C. C. 1998. SPARC deficiency leads to early-onset cataractogenesis. Invest. Ophthalmol. Vis. Sci. 39: 2674-2680. [Medline]

17. Rentz, T. J., Poobalarahi, F., Bornstein, P., Sage, E. H. and Bradshaw, A. D. 2007. SPARC regulates processing of procollagen I and collagen fibrillogenesis in dermal fibroblasts. J. Biol. Chem. 282: 22062-22071. [Medline] [CrossRef]

18. Schellings, M. W., Vanhoutte, D., Swinnen, M., Cleutjens, J. P., Debets, J., van Leeuwen, R. E., d'Hooge, J., Van de Werf, F., Carmeliet, P., Pinto, Y. M., Sage, E. H. and Heymans, S. 2009. Absence of SPARC results in increased cardiac rupture and dysfunction after acute myocardial infarction. $J$. Exp. Med. 206: 113-123. [Medline] [CrossRef]

19. Trensz, F., Lucien, F., Couture, V., Söllrald, T., Drouin, G., Rouleau, A. J., Grandbois, M., Lacraz, G. and Grenier, G. 2015. Increased microenvironment stiffness in damaged myofibers promotes myogenic progenitor cell proliferation. Skelet. Muscle 5: 5. [Medline] [CrossRef] 